J Neurol Neurosurg Psychiatry. 2016 August ; 87(8): 817-820. doi:10.1136/jnnp-2015-311438.

\title{
Traumatic Brain Injury History is Associated with Earlier Age of Onset in Frontotemporal Dementia
}

\author{
Christian LoBue ${ }^{1}$, Kristin Wilmoth ${ }^{1}$, C. Munro Cullum ${ }^{1,2}$, Heidi C. Rossetti ${ }^{1}$, Laura H. \\ Lacritz $^{1,2}$, Linda S. Hynan ${ }^{1,3}$, John Hart Jr. ${ }^{1,2,4}$, and Kyle B. Womack ${ }^{1,2,4}$ \\ ${ }^{1}$ Department of Psychiatry, University of Texas Southwestern Medical Center, Dallas, TX, USA \\ ${ }^{2}$ Department of Neurology and Neurotherapeutics, University of Texas Southwestern Medical \\ Center, Dallas, TX, USA \\ ${ }^{3}$ Department of Clinical Sciences, University of Texas Southwestern Medical Center, Dallas, TX, \\ USA \\ ${ }^{4}$ Center for BrainHealth, School of Behavioral and Brain Sciences, University of Texas at Dallas, \\ Dallas, TX, USA
}

\begin{abstract}
Objective-We retrospectively examined whether a history of traumatic brain injury (TBI) is associated with an earlier age of symptom onset and diagnosis in a large sample of patients with behavioral variant frontotemporal dementia (bvFTD).

Methods-Subjects with bvFTD ( $\mathrm{n}=678$ ) were obtained from the National Alzheimer's Coordinating Center Uniform Data Set. TBI was categorized based on reported lifetime history of TBI with loss of consciousness (LOC) but no chronic deficits occurring more than one year prior to diagnosis of bvFTD. ANCOVA was used to determine if clinician-estimated age of symptom onset and age at diagnosis of bvFTD differed between those who reported a history of TBI with LOC (TBI+) and those who did not (TBI-).
\end{abstract}

Results-Controlling for sex, the TBI+ bvFTD group had an earlier age of symptom onset and age of diagnosis that was on average 2.8 and 3.2 years earlier (p's<.01) than the TBI- bvFTD group.

Conclusion-TBI history with LOC occurring more than one year prior to diagnosis is associated with an earlier age of symptom onset and diagnosis in patients with bvFTD. TBI may be related to the underlying neurodegenerative processes in bvFTD, but the implications of age at time of injury, severity, and repetitive injuries remain unclear.

\section{Keywords}

National Alzheimer's Coordinating Center (NACC); Traumatic Brain Injury (TBI); Dementia; Frontotemporal Dementia (FTD); Age of Onset

*Corresponding Author: Kyle B. Womack, M.D., 5323 Harry Hines Blvd., Dallas, TX 75208-8564, 214-648-0373 (phone); 214-648-2031 (fax), kyle.womack@utsouthwestern.edu.

Competing Interests: The authors disclose no conflicts of interest. 
Traumatic brain injury (TBI) has garnered support as a risk factor for the later development of dementia,[1-7] but not all studies report such an association.[8-10] A recent retrospective analysis of electronic medical records compared older subjects with a diagnosis of TBI within 5-7 years $(n=51,799)$ to subjects without any history of TBI $(n=122,862)$ and found that those with moderate-to-severe TBI were at a higher risk of developing dementia.[2] In another retrospective chart review among Swedish males drafted into military service, those with a past diagnosis of moderate-severe TBI were at a higher risk of developing dementia. [1] While these findings suggest that TBI may be a risk factor for later cognitive decline, risk factors may play different roles across various types of dementia.[11] Alzheimer Disease (AD) is the most common type of dementia, constituting approximately $42-77 \%$ of neuropathologically confirmed cases,[12 13] followed by dementia with lewy bodies [(DLB); 26\%],[13] vascular dementia (18-24\%),[12 13] and frontotemporal dementia [(FTD); 3-6\%].[12 13] Whereas a history of moderate to severe TBI has been associated with a higher risk of developing AD in several investigations, [3 5 7] there is limited evidence on the magnitude of risk associated with TBI in other types of dementia. This may be due to the relative infrequency of non-AD cases, [3] but may also be related to methodological factors such as reliance upon medical record diagnostic codes outside of specialized dementia clinics. To date, we know of only three studies that have specifically investigated the association between a history of TBI and FTD. First, Rosso et al. (2003) compared 80 patients with a bvFTD diagnosis to 124 demographically matched controls and reported that a history of TBI of any severity was associated with a 3.3 higher risk of a diagnosis of behavioral variant FTD (bvFTD).[14] In a follow-up study, Kalkonde et al. (2012) compared 63 veterans with a bvFTD diagnosis to 491 veterans with a non-FTD dementia diagnosis and reported that a history of TBI with loss of consciousness (LOC) was associated with a 4.4 higher risk of bvFTD.[15] Further, Deutsch et al., (2015) recently conducted a retrospective investigation comparing a large sample of patients with an FTD diagnosis $(n=1,016)$ to controls $(n=2,015)$ and found that a lifetime history of TBI with $>5$ minutes loss of consciousness (mLOC) was associated with a 1.6 higher risk for FTD diagnosis.[16]

In contrast to the above mentioned neurodegenerative disorders, chronic traumatic encephalopathy (CTE) is defined by its association with TBI. The concept of CTE, although under different names, has been in the literature at least since Martland's 1928 clinical case report of a professional boxer who developed dementia[17] and has been intimately tied to the presence of repetitive TBI in both the clinical and pathological literature ever since.[18] The neuropathological findings in CTE are well described.[19-21] While these are distinct from other degenerative dementias in form and distribution, some commonality to FTD exists in the species of proteins that aggregate. CTE is primarily characterized by neurofibrillary tangles comprised of tau; however, TAR DNA-binding protein (TDP-43) inclusions have accompanied these in some case descriptions.[1921] The majority of FTD syndromes, including bvFTD, show pathological substrates involving either TDP-43 (50\%) or tau (40\%).[22] Although a direct mechanistic link between TBI and these pathological processes is lacking, some evidence suggests that these may be related. In nearly all severities of acute TBI, axonal tracts stretch, shear, and compress, often resulting in diffuse axonal injury.[23] Biochemical functions and cytoskeletal structures become compromised 
and lead to axonal swelling which may contribute to a gradual degenerative process in neurons and/or neuronal transmission.[23 24] It is hypothesized that because of diffuse axonal injury, neuronal alterations might disrupt the regulation of tau and contribute to abnormal tau aggregation at some point following injury. For example, tau pathology was absent acutely after a single, severe TBI in one study[25] but was reported after at least 1 year following such an injury in another investigation.[24] Further, persons with a history of a single TBI had more widespread tau aggregation and at a much earlier age than agematched controls.[24] With regard to TDP-43, this association is less clear. In pathological inclusions, TDP-43 is characteristically hyperphosphorylated and translocated from the nucleus to the cytoplasm. Such TDP-43 inclusions have been described in addition to tau pathology in some cases of CTE that have a history of recurrent TBI.[19] One investigation that examined TDP-43 pathology in patients with a single moderate/severe TBI found increased cytoplasmic TDP-43 but it was not pathologically hyperphosphorylated.[26] However, nearly all cases of amyotrophic lateral sclerosis (ALS), a neurodegenerative motor neuron disease of the central nervous system, are found to have TDP-43 pathology and some studies have reported that patients with repetitive TBI have a higher risk for later developing ALS.[27 28] Such findings suggest that TBI may be a neurodegenerative risk factor via repercussions on tau and TDP-43 pathophysiology, although these associations remain poorly understood.

The implications of a TBI history in the later development of dementia may extend beyond higher absolute risk and also include an earlier age of onset. For example, a recent investigation showed that a history of TBI was associated with a 2.1 year earlier onset of dementia in a large sample $(n=196)$.[3] Earlier onset has been observed separately in small samples of TBI subjects who later developed $\mathrm{AD}[29$ 30] although the literature has been mixed.[6 31] A recent analysis of a large sample of patients with a diagnosis of mild cognitive impairment (MCI) revealed an association between a history of TBI with LOC and increased risk of MCI as well as an approximate two year earlier onset (LoBue et al., under review*).

Thus, mounting evidence suggests that a history of TBI may confer an increased risk of later-life cognitive impairment and decline, although whether or not an earlier age of onset occurs in bvFTD is less clear. Only one recent study has examined this association in bvFTD by comparing 27 subjects with a lifetime history of TBI of $>5 \mathrm{mLOC}$ to those without ( $\mathrm{n}$ $>600$ ) and found that age of onset was similar among the groups. A non-association with TBI in that study may have been related to the limited number of TBI cases or to the inclusion of milder $(<5 \mathrm{mLOC})$ TBIs in the comparison group. The aim of this study was to examine whether a reported history of TBI with LOC of any duration was associated with an earlier age of symptom onset and diagnosis in a large cohort of individuals with a diagnosis of bvFTD. Such findings could support an emerging literature that TBI may play a role in the development of tau-related and possibly TDP-43 related dementias other than CTE.

\section{Methods}

Since September, 2005 the National Alzheimer's Coordinating Center (NACC) has maintained an integrated database of information pooled from 34 past and present National 
Institute of Aging (NIA) - funded Alzheimer's Disease Centers (ADC) across the U.S. Sociodemographic and clinical information were selected from the NACC Uniform Data Set (UDS)[32] for subjects over age 40 with a diagnosis of bvFTD with initial visits completed between September, 2005 and December, 2013. Diagnosis was determined by ADC clinicians using standard diagnostic guidelines which included Neary criteria.[33] For the purpose of the present study, the age subjects' were diagnosed with bvFTD at the initial ADC visit, education, sex, race, first-degree family history of dementia, and clinicianestimated age of cognitive decline (hereafter referred to as symptom onset) at initial visit were examined. Only subjects with complete data were included in the present study.

At the initial ADC visit, subjects answered three standard questions in the NACC database related to TBI regarding whether they had ever sustained a TBI resulting in $<5$ minutes loss of consciousness (mLOC), $\geq 5 \mathrm{mLOC}$, and whether there was a chronic neurological deficit/ dysfunction as a result of the injury. Each question was answered as absent, recent/active (defined as occurring within 1 year of visit or currently requiring treatment), remote/inactive (defined as occurring $>1$ year of visit and either having recovered from the injury or there is no current treatment underway), or unknown. As the purpose of this study was to examine a possible association between a history of TBI and onset of bvFTD, we attempted to limit potential confounding for neurological decline that may be directly attributable to a TBI. Those individuals with a diagnosis of bvFTD who reported either a remote ( $>1$ year of visit) TBI with LOC and no chronic deficits or an absence of TBI history prior to their initial ADC visit were included. In contrast, subjects who reported a recent/active TBI $[<1$ year of visit, $\mathrm{n}=10$ ] or any history of TBI resulting in chronic deficit/dysfunction $(\mathrm{n}=5)$ at their initial visit were excluded.

\section{Statistical Analysis}

Chi-square was used to compare sex, race, and family history of dementia between those with a self-reported history of TBI (TBI+) and those without (TBI-) in the bvFTD sample. Independent t-tests assessed whether education differed between the groups. Variables that significantly differed between the groups were entered as covariates into primary analyses. ANCOVAs were used to determine if estimated age of symptom onset and age at diagnosis differed between the TBI+ and TBI- bvFTD groups. All analyses were conducted using IBM $^{\odot}$ SPSS Statistics V22 (IBM Corp, SPSS Statistics V22, Armonk, NY, 2013) with $p<$. 05 as the level for significance. Assumptions for all tests were reviewed.

\section{Results}

\section{Clinical, Demographic, and Sample Characteristics}

bvFTD Sample-A total of 678 subjects diagnosed with bvFTD met inclusion criteria. Among these, 75 reported a history of TBI with LOC and 603 subjects did not. ADC clinicians recognized TBI as possibly contributing to a dementia diagnosis in only 2 cases. The TBI+ and TBI- groups did not differ by race $(p=.17)$, family history of dementia ( $p=$. 93 ), or education $[p=.61$, Table 1$]$. Both groups were predominantly Caucasian $(>90 \%)$, had $51 \%$ of subjects with a family history of dementia, and were well-educated (14-15 mean 
years of education). The bvFTD groups did differ in terms of sex distribution $(p<.001)$, with the TBI+ group being $17 \%$ female while the TBI- group was $39 \%$ female.

\section{TBI History on Estimated Age of Symptom Onset and Age of Diagnosis}

Controlling for sex, estimated age of symptom onset was on average 2.8 years earlier $(p=$. $005)$ for the TBI+ $\left(\mathrm{M}_{\mathrm{Age}}=56.2, \mathrm{SD}=8.2\right)$ bvFTD group compared to the TBI- group $\left[\mathrm{M}_{\mathrm{Age}}\right.$ $=59.0, \mathrm{SD}=9.1$, Table 2]. Similarly, the TBI+ group $\left(\mathrm{M}_{\mathrm{Age}}=61.5, \mathrm{SD}=8.0\right)$ was diagnosed with bvFTD on average 3.2 years earlier $(p=.002)$ than the TBI- group $\left(\mathrm{M}_{\text {Age }}=\right.$ 64.7, $\mathrm{SD}=9.0$ ). A history of TBI demonstrated a small-to-medium effect size for estimated age of symptom onset $(d=.32)$ and age of diagnosis $(d=.37)$ in bvFTD (see Table 1$)$.

\section{Death and Neuropathological Data}

Among the 678 participants with a bvFTD diagnosis at the initial visit, 137 later died and came to autopsy. Of those, 9 had a history of TBI with LOC occurring more than one year prior to the initial visit. Primary neuropathological diagnoses were as follows: FTLD ( $n_{\text {TBI+ }}$ $\left.=6, \mathrm{n}_{\mathrm{TBI}}=92\right), \mathrm{AD}\left(\mathrm{n}_{\mathrm{TBI}+}=3, \mathrm{n}_{\mathrm{TBI}}=29\right)$, vascular disease $\left(\mathrm{n}_{\mathrm{TBI}+}=0, \mathrm{n}_{\mathrm{TBI}}=2\right)$, Lewy Body disease $\left(\mathrm{n}_{\mathrm{TBI}+}=0, \mathrm{n}_{\mathrm{TBI}}=3\right)$, prion-related disease $\left(\mathrm{n}_{\mathrm{TBI}+}=0, \mathrm{n}_{\mathrm{TBI}}=1\right)$, and hippocampal sclerosis $\left(\mathrm{n}_{\mathrm{TBI}+}=0, \mathrm{n}_{\mathrm{TBI}}=1\right)$.

\section{Discussion}

A history of TBI with LOC was associated with an earlier age of symptom onset and age of diagnosis in bvFTD (i.e., 2.8 and 3.2 years respectively), and these effects were independent of family history of dementia and education. Although a larger percentage of males were found to be in the TBI+ group than the TBI- group, this finding is generally consistent with the TBI literature.[34] This study is the first to provide preliminary evidence suggesting that TBI may be a risk factor for earlier expression of bvFTD later in life. This is in contrast to a previous report that also compared bvFTD subjects from the NACC database and found no difference in age of bvFTD onset in a small number of subjects with a history of TBI of $>5 \mathrm{mLOC}(\mathrm{n}<30)$ compared to those without such a history.[16] One simple explanation for this difference may be related to the increased statistical power in the present study due to a larger number of TBI cases ( $n=75$ vs $n<30$ ). To further explore this issue, we conducted a post-hoc analysis similar to Deutsche et al. (2015) and excluded TBI+ subjects with $<5$ mLOC. A one-way ANCOVA controlling for sex similarly failed to show that age of symptom onset differed $(p=.45)$ among TBI+ subjects with $\geq 5 \mathrm{mLOC}\left(\mathrm{n}=24 ; \mathrm{M}_{\mathrm{Age}}=\right.$ $57.8)$ and those without any history of TBI $\left(n=603 ; M_{\text {Age }}=59.0\right)$. This suggests that decreased statistical power in the study by Deutsche et al. (2015) accounted for the lack of association between a history of TBI and age of symptom onset in bvFTD subjects. Only three studies to date have investigated the associated risk between TBI and bvFTD, and when these are combined with the current findings, mounting evidence indicates that TBI with LOC may be a risk factor in bvFTD.

Neurodegenerative dementias such as bvFTD have an insidious onset, typically unfolding over many years. The biological damage involved in neurodegeneration progresses in a latent phase until a threshold is reached, at which point cognitive/behavioral impairments 
become clinically manifest.[35] As such, by the time dementia is diagnosed, the time between onset of the biological damage and its clinical manifestation may be many years apart. TBI, even at a milder severity, is known to cause neuronal and axonal injury[36 37] and result in a cascade of abnormal neurochemical processes.[38] Along these lines, anatomically, bvFTD involves degeneration primarily in the frontal lobes and the anterior temporal regions,[39] and it is worth noting that TBI has a well-known predilection for affecting these regions and their underlying connections. [40] While it is unclear how the effects of TBI may play a role in a later neurodegenerative process, based on the present findings and other evidence, it appears that TBI with LOC may reduce the time needed to reach the threshold for clinical manifestation in some individuals with bvFTD.

Ever since Martland's first case report of a professional boxer in 1928, a link between repetitive TBI and CTE has become established.[19 20] Given this association, it would appear plausible that other neurodegenerative disorders/dementias could also be linked to TBI for some individuals, which is supported by emerging literature suggesting TBI is associated with increased risk for and earlier onset of the development of all-cause dementia, [2-4] AD,[5-7 30] and ALS,[27 28] as well as in FTD. While the pathology of CTE, AD, ALS, FTD and other neurodegenerative disorders are distinct, the involvement of TDP-43 and/or tau overlaps these disorders and others. Therefore, the mechanisms that cause CTE by impacting TDP-43 and tau may also cause other related neurodegenerative disorders as well, including FTD.

Several methodologic limitations may restrict generalizability of our findings. The lack of TBI details regarding age at the time of TBI, severity, repetitive injuries, etc. in the NACC database did not allow potentially important variables to be examined that may play a role in the neurodegenerative process. It should also be noted that the arbitrary cutoff of $<5$ and $\geq 5$ $\mathrm{mLOC}$ for the TBI criteria in the NACC dataset is unclear and inconsistent with most conventional criteria for TBI classification. Another issue is the time since TBI. Based on the definition for "remote" TBI, injuries may have occurred as recently as nearly one year prior to the initial visit, which may limit conclusions regarding injuries that occurred decades earlier. Falls are one of the leading causes of TBI in an aging population, and contribute to higher rates of TBI-related hospitalizations in individuals 65 and over.[34] Thus, an unknown proportion of TBIs in this sample could have been relatively more "recent," and possibly occurred following onset of a dementia process, especially given that the neuropathological processes underlying dementia often precedes its clinical manifestation by several years. Therefore, "remote" TBI is a relative term and further investigation is needed along these lines. Last, the limited neuropathology data revealed that other dementia types were present in our sample, and although FTLD pathology was found in $66 \%$ of TBI+ and $72 \%$ of TBI- cases that went to autopsy, it is unclear whether a study design using autopsy-confirmed cases would yield similar results.

Despite limitations in the available data, current results suggest that a history of TBI with LOC occurring more than one year prior to diagnosis is associated with an earlier age of symptom onset and diagnosis in patients with bvFTD. These findings support emerging literature of TBI as a risk factor for cognitive decline later in life including tau- and TDP-43related dementias. Future research should incorporate questionnaires that more thoroughly 
assess TBI characteristics (e.g. severity, age of injury, presence and duration of LOC, posttraumatic symptoms, etc.) as well as other neurobiological phenotypes and genotypes in order to identify which factors may be most associated with earlier onset dysfunction associated with bvFTD and related disorders.

\section{Acknowledgments}

The NACC database is funded by NIA/NIH Grant U01 AG016976. NACC data are contributed by the NIA funded: P30 AG019610 (PI Eric Reiman, MD), P30 AG013846 (PI Neil Kowall, MD), P50 AG008702 (PI Scott Small, MD), P50 AG025688 (PI Allan Levey, MD, PhD), P30 AG010133 (PI Andrew Saykin, PsyD), P50 AG005146 (PI Marilyn Albert, PhD), P50 AG005134 (PI Bradley Hyman, MD, PhD), P50 AG016574 (PI Ronald Petersen, MD, PhD), P50 AG005138 (PI Mary Sano, PhD), P30 AG008051 (PI Steven Ferris, PhD), P30 AG013854 (PI M. Marsel Mesulam, MD), P30 AG008017 (PI Jeffrey Kaye, MD), P30 AG010161 (PI David Bennett, MD), P30 AG010129 (PI Charles DeCarli, MD), P50 AG016573 (PI Frank LaFerla, PhD), P50 AG016570 (PI David Teplow, PhD), P50 AG005131 (PI Douglas Galasko, MD), P50 AG023501 (PI Bruce Miller, MD), P30 AG035982 (PI Russell Swerdlow, MD), P30 AG028383 (PI Linda Van Eldik, PhD), P30 AG010124 (PI John Trojanowski, MD, PhD), P50 AG005133 (PI Oscar Lopez, MD), P50 AG005142 (PI Helena Chui, MD), P30 AG012300 (PI Roger Rosenberg, MD), P50 AG005136 (PI Thomas Montine, MD, PhD), P50 AG033514 (PI Sanjay Asthana, MD, FRCP), and P50 AG005681 (PI John Morris, MD), P50 AG000000 (PI Bruce Wayne and Co-PI Richard Grayson, Gotham City Concussion Study) . The Alzheimer's Disease Genetic Consortium (ADGC) is funded by NIA Grant U01 AG032984.

Source of Funding: Funding for this study was provided in part by the NIH/NIA P30 AG012300 Alzheimer's Disease Center Grant.

\section{References}

1. Nordström P, Michaëlsson K, Gustafson Y, et al. Traumatic brain injury and young onset dementia: a nationwide cohort study. Annals of neurology. 2014; 75:374-81. [PubMed: 24812697]

2. Gardner RC, Burke JF, Nettiksimmons J, et al. Dementia risk after traumatic brain injury vs nonbrain trauma: the role of age and severity. JAMA neurology. 2014; 71:1490-97. [PubMed: 25347255]

3. Barnes DE, Kaup A, Kirby KA, et al. Traumatic brain injury and risk of dementia in older veterans. Neurology. 2014; 83:312-19. [PubMed: 24966406]

4. Wang H-K, Lin S-H, Sung P-S, et al. Population based study on patients with traumatic brain injury suggests increased risk of dementia. Journal of Neurology, Neurosurgery \& Psychiatry. 2012; 83:1080-85.

5. Fleminger S, Oliver D, Lovestone S, et al. Head injury as a risk factor for Alzheimer's disease: the evidence 10 years on; a partial replication. Journal of Neurology, Neurosurgery \& Psychiatry. 2003; 74:857-62.

6. Plassman BL, Havlik R, Steffens D, et al. Documented head injury in early adulthood and risk of Alzheimer's disease and other dementias. Neurology. 2000; 55:1158-66. [PubMed: 11071494]

7. Guo Z, Cupples L, Kurz A, et al. Head injury and the risk of AD in the MIRAGE study. Neurology. 2000; 54:1316-23. [PubMed: 10746604]

8. Helmes E, Østbye T, Steenhuis RE. Incremental contribution of reported previous head injury to the prediction of diagnosis and cognitive functioning in older adults. Brain injury. 2011; 25:338-47. [PubMed: 21314278]

9. Lindsay J, Laurin D, Verreault R, et al. Risk factors for Alzheimer's disease: a prospective analysis from the Canadian Study of Health and Aging. American Journal of Epidemiology. 2002; 156:44553. [PubMed: 12196314]

10. Dams-O'Connor K, Gibbons LE, Bowen JD, et al. Risk for late-life re-injury, dementia and death among individuals with traumatic brain injury: a population-based study. Journal of Neurology, Neurosurgery \& Psychiatry. 2013; 84:177-82.

11. Boot BP, Orr CF, Ahlskog JE, et al. Risk factors for dementia with Lewy bodies: a case-control study. Neurology. 2013; 81:833-40. [PubMed: 23892702] 
12. Brunnström H, Gustafson L, Passant U, et al. Prevalence of dementia subtypes: a 30-year retrospective survey of neuropathological reports. Archives of gerontology and geriatrics. 2009; 49:146-9. [PubMed: 18692255]

13. Barker WW, Luis CA, Kashuba A, et al. Relative frequencies of Alzheimer disease, Lewy body, vascular and frontotemporal dementia, and hippocampal sclerosis in the State of Florida Brain Bank. Alzheimer Disease \& Associated Disorders. 2002; 16:203-12. [PubMed: 12468894]

14. Rosso S, Landweer E, Houterman M, et al. Medical and environmental risk factors for sporadic frontotemporal dementia: a retrospective case-control study. Journal of Neurology, Neurosurgery \& Psychiatry. 2003; 74:1574-76.

15. Kalkonde YV, Jawaid A, Qureshi SU, et al. Medical and environmental risk factors associated with frontotemporal dementia: a case-control study in a veteran population. Alzheimer's \& Dementia. 2012; 8:204-10.

16. Deutsch MB, Mendez MF, Teng E. Interactions between traumatic brain injury and frontotemporal degeneration. Dementia and geriatric cognitive disorders. 2015; 39:143-53. [PubMed: 25531628]

17. Martland HS. Punch drunk. Journal of the American Medical Association. 1928; 91:1103-07.

18. Corsellis J, Brierley J. Observations on the pathology of insidious dementia following head injury. The British Journal of Psychiatry. 1959; 105:714-20.

19. McKee AC, Gavett BE, Stern RA, et al. TDP-43 proteinopathy and motor neuron disease in chronic traumatic encephalopathy. Journal of neuropathology and experimental neurology. 2010; 69:918-29. [PubMed: 20720505]

20. McKee AC, Cantu RC, Nowinski CJ, et al. Chronic traumatic encephalopathy in athletes: progressive tauopathy following repetitive head injury. Journal of neuropathology and experimental neurology. 2009; 68:709-35. [PubMed: 19535999]

21. McKee AC, Stein TD, Nowinski CJ, et al. The spectrum of disease in chronic traumatic encephalopathy. Brain. 2013; 136:43-64. [PubMed: 23208308]

22. Ng AS, Rademakers R, Miller BL. Frontotemporal dementia: a bridge between dementia and neuromuscular disease. Annals of the New York Academy of Sciences. 2014; 1338:71-93. [PubMed: 25557955]

23. Johnson VE, Stewart W, Smith DH. Axonal pathology in traumatic brain injury. Experimental neurology. 2013; 246:35-43. [PubMed: 22285252]

24. Johnson VE, Stewart W, Smith DH. Widespread tau and amyloid-beta pathology many years after a single traumatic brain injury in humans. Brain Pathology. 2012; 22:142-9. [PubMed: 21714827]

25. Smith C, Graham D, Murray L, et al. Tau immunohistochemistry in acute brain injury. Neuropathology and applied neurobiology. 2003; 29:496-502. [PubMed: 14507341]

26. Johnson VE, Stewart W, Trojanowski JQ, et al. Acute and chronically increased immunoreactivity to phosphorylation-independent but not pathological TDP-43 after a single traumatic brain injury in humans. Acta neuropathologica. 2011; 122:715-26. [PubMed: 22101322]

27. Chen H, Richard M, Sandler DP, et al. Head injury and amyotrophic lateral sclerosis. American journal of epidemiology. 2007; 166:810-16. [PubMed: 17641152]

28. Pupillo E, Messina P, Logroscino G, et al. Trauma and amyotrophic lateral sclerosis: a case-control study from a population-based registry. European Journal of Neurology. 2012; 19:1509-17. [PubMed: 22537412]

29. Sullivan P, Petitti D, Barbaccia J. Head trauma and age of onset of dementia of the Alzheimer type. Jama. 1987; 257:2289-90. [PubMed: 3573227]

30. Nemetz PN, Leibson C, Naessens JM, et al. Traumatic brain injury and time to onset of Alzheimer's disease: a population-based study. American Journal of Epidemiology. 1999; 149:3240. [PubMed: 9883791]

31. Rasmusson D, Brandt J, Martin D, et al. Head injury as a risk factor in Alzheimer's disease. Brain Injury. 1995; 9:213-9. [PubMed: 7606235]

32. Morris JC, Weintraub S, Chui HC, et al. The Uniform Data Set (UDS): clinical and cognitive variables and descriptive data from Alzheimer Disease Centers. Alzheimer Disease \& Associated Disorders. 2006; 20:210-6. [PubMed: 17132964]

33. Neary D, Snowden JS, Gustafson L, et al. Frontotemporal lobar degeneration: a consensus on clinical diagnostic criteria. Neurology. 1998; 51:1546-54. [PubMed: 9855500] 
34. Faul, M.; Xu, L.; Wald, MM., et al. Traumatic brain injury in the United States: emergency department visits, hospitalizations, and deaths 2002 - 2006. Vol. 2. Atlanta (GA): Centers for Disease Control and Prevention National Center for Injury Prevention and Control; 2010. p. 1-9.

35. Chapman J, Korczyn AD, Karussis DM, et al. The effects of APOE genotype on age at onset and progression of neurodegenerative diseases. Neurology. 2001; 57:1482-5. [PubMed: 11673593]

36. Allen MD, Wu TC, Bigler ED. Traumatic brain injury alters Word Memory Test performance by slowing response time and increasing cortical activation: an fMRI study of a symptom validity test. Psychological Injury and Law. 2011; 4:140-6.

37. Messé A, Caplain S, Paradot G, et al. Diffusion tensor imaging and white matter lesions at the subacute stage in mild traumatic brain injury with persistent neurobehavioral impairment. Human brain mapping. 2011; 32:999-1011. [PubMed: 20669166]

38. Hovda D, Lee S, Smith M, et al. The neurochemical and metabolic cascade following brain injury: moving from animal models to man. Journal of neurotrauma. 1995; 12:903-6. [PubMed: 8594218]

39. Schoenberg, MR.; Duff, K. Dementias and mild cognitive impairment in adults. In: Schoenberg, MR.; Scott, JG., editors. The Little Black Book of Neuropsychology: A Syndrome-Based Approach. New York, NY: Springer; 2011. p. 357-403.

40. Zappalà G, Thiebaut de Schotten M, Eslinger PJ. Traumatic brain injury and the frontal lobes: what can we gain with diffusion tensor imaging? Cortex. 2012; 48:156-65. [PubMed: 21813118] 
Table 1

Demographic and Clinical Characteristics of bvFTD Subjects With and Without TBI

\begin{tabular}{|c|c|c|c|}
\hline & $\begin{array}{c}T B I+ \\
(n=75)\end{array}$ & $\begin{array}{c}T B I- \\
(n=603)\end{array}$ & p-value \\
\hline Education in years, $M[S D]$ & $14.7[2.8]$ & $14.9[3.3]$ & .61 \\
\hline Age of Diagnosis in years, $M[S D]$ & $61.5[8.0]$ & $64.7[9.0]$ & .002 \\
\hline Estimated Age of Symptom Onset in years, $M[S D]$ & $56.2[8.2]$ & $59.0[9.1]$ & .005 \\
\hline Female, n (\%) & $13(17 \%)$ & $235(39 \%)$ & $<.001$ \\
\hline Non-Hispanic Caucasian, n (\%) & $74(99 \%)$ & $561(93 \%)$ & .17 \\
\hline Family history of Dementia, $\mathrm{n}(\%)$ & $38(51 \%)$ & $308(51 \%)$ & .93 \\
\hline
\end{tabular}

Note. TBI+ are subjects with history of traumatic brain injury (TBI) with loss of consciousness; TBI- are subjects without TBI. Age of Diagnosis reflects subjects' age at the initial visit when a diagnosis of bvFTD was received. 